\title{
Die Form des Wirtschaftens ändern
}

\section{Mit dem Sozialstaatsprinzip zu Vergesellschaftungen}

Zusammenfassung:Vergesellschaftungen sind nicht mehr eine abstrakte Angelegenheit, ihre Notwendigkeit wird immer auffälliger. Vergesellschaftungen sind eine Form der Umsetzung des Sozialstaatsprinzips und nicht mit Enteignungen zu verwechseln. Es ist ein politischer und juristischer Kampf, Vergesellschaftungen spruchreif zu machen.

Schlagwörter: Vergesellschaftung, Sozialstaat, Enteignung

\section{Changing the way we perform economic activity} Socializing by using the welfare state principle

Abstract: Socializations are no longer an abstract matter, their necessity becomes more and more obvious. Socializations are a form of implementing the welfare state principle and should not be confused with expropriations. It is a political and legal struggle to make socializations ripe for decision.

Keywords: Socialization, welfare state, expropriation

$\mathrm{M}$ it der Initiative $»$ Deutsche Wohnen enteignen « in Berlin, die die Vergesellschaftung großer Wohnungsunternehmen erreichen will, wurden Vergesellschaftungen erstmals zu einem realistischen Szenario in der Politik. Die Corona-Krise zeigt, dass insbesondere im Gesundheitssektor ebenfalls über Vergesellschaftungen nachzudenken ist, denn der Markt regelt jedenfalls nicht, dass die Bedürfnisse befriedigt werden können. Wohlgemerkt, Verge-

* Halina Wawzyniak ist Volljuristin und war in den Jahren 2009 bis 2017 Abgeordnete des Deutschen Bundestages. sellschaftungen, nicht Enteignungen. Der Unterschied scheint auf den ersten Blick marginal zu sein. Tatsächlich sind Enteignung und Vergesellschaftung jedoch zwei komplett unterschiedliche Konzepte. Das ist keine rein juristische Frage, sondern auch eine politische.

Vergesellschaftungen sind kein Selbstzweck. Wenn über Vergesellschaftungen gesprochen wird, dann geht es um die Erfüllung des Sozialstaatsgedankens des Grundgesetzes. Nicht alles kann oder sollte vergesellschaftet werden, aber wenn es um die Daseinsvorsorge geht und der Markt nachweislich keine Lösung bietet, müssen 
sie thematisiert werden. Hierfür bedarf es einer politischen Debatte und Sensibilisierung sowie juristischer Unterfütterung- denn so einfach ist das mit der Vergesellschaftung nämlich nicht.

\section{Der Sozialstaat}

Es ist ein unscheinbarer Halbsatz im Grundgesetz (GG), der das Sozialstaatsprinzip begründet. Dieser Halbsatz ist neben Artikel 15 GG eine Grundlage zur Begründung von Vergesellschaftungen. Das wird in den politischen Debatten häufig übersehen - und erst recht in den juristischen. Der Artikel 20 Abs. 1 GG lautet: »Die Bundesrepublik Deutschland ist ein demokratischer und sozialer Bundesstaat.« Diese Formulierung stellt nach der herrschenden juristischen Meinung eine Staatszielbestimmung dar. Dies meint, dass alle Staatsorgane verpflichtet sind, das Ziel eines sozialen Bundesstaates zu verfolgen. Ihr Handeln muss also auf die Gewährleistung des Sozialstaates orientieren. Gesetzliche Regelungen sind immer auch unter dem Gesichtspunkt zu betrachten, ob sie der Erfüllung des Staatsziels dienen. Was diesen Sozialstaat aber nun ausmacht, was elementare Bestandteile des Sozialstaates sind, das ist wiederum eine politische Debatte. In linken Diskursen wird häufig kritisiert, dass eine Staatszielbestimmung nicht mit einem konkret einklagbaren Anspruch auf bestimmte staatliche Leistungen verbunden ist. Um diese Kritik soll es im Folgenden aber nicht gehen.

Für eine politische Auseinandersetzung ist relevant, dass mit der Staatszielbestimmung der Weg für den Gesetzgeber frei ist, gut begründet dar- zulegen, warum bestimmte Mittel zur Erreichung des Staatszieles notwendig sind - also zum Beispiel Vergesellschaftungen. Unbestritten zählen zu den Grundelementen des Sozialstaatsprinzips, dass es einen Anspruch auf soziale Sicherheit und sozialen Ausgleich geben muss, der aus dem Solidaritätsgedanken erwächst. Und das Sozialstaatsprinzip umfasst Hilfe gegen Not und Armut ebenso, wie den Anspruch auf ein menschenwürdiges Existenzminimum. Zum Sozialstaat gehört die Daseinsvorsorge, also die Versorgung mit lebensnotwendigen Gütern und Dienstleistungen - und das sind eben auch Krankenhäuser, Wohnraum und ein Zugang zu Kommunikationsmitteln. Artikel 20 GG schreibt »das Soziale als ebenso legitime wie obligatorische Staatsaufgabe vor " (Dreier 2016, Art. 20, Rdnr. 24). Und die Verpflichtung aus dieser Staatsaufgabe ist dann, dass die Institutionen "soziale Verwerfungen nicht wie der (allzu) sprichwörtliche `Nachtwächterstaat` ignorieren", sondern den Auftrag haben, "sowohl zum (reaktiven) Gegensteuern als auch zum (aktiven) Gestalten der sozialen Ordnung" (ebd.). Die Ausgestaltung der sozialen Ordnung im Rahmen des Sozialstaates wiederum ist Aufgabe der Politik.

Die Verankerung des Sozialstaatsprinzips im Grundgesetz ist keine Selbstverständlichkeit. Dies zeigte bereits die in den 1950er Jahren geführte Debatte zwischen dem Staatsrechtler Ernst Forsthoff und Politikwissenschaftlers Wolfgang Abendroth zum Sozialstaatsbegriff. Im Konvent von Herrenchiemsee beispielsweise gab es keine Sozialstaatsklausel (vgl. Dreier 2016, Art. 20, 
Rdnr. 10). Der Artikel 20 GG steht unter der sogenannten Ewigkeitsgarantie, er ist also auch mit einer verfassungsändernden Mehrheit nicht abzuschaffen oder zu verändern.

Das alles zusammengenommen ergibt für politisches Handeln erhebliche Spielräume. Zumal das Bundesverfassungsgericht (BVerfGE) in einer grundlegenden Entscheidung aus dem Jahr 2010 ein Grundrecht auf Gewährleistung eines menschenwürdigen Existenzminimums anerkannt hat: "Das Grundrecht auf Gewährleistung eines menschenwürdigen Existenzminimums aus Art. 1 Abs. 1 GG in Verbindung mit dem Sozialstaatsprinzip des Art. 20 Abs. 1 GG sichert jedem Hilfebedürftigen diejenigen materiellen Voraussetzungen $\mathrm{zu}$, die für seine physische Existenz und für ein Mindestmaß an Teilhabe am gesellschaftlichen, kulturellen und politischen Leben unerlässlich sind." (BVerfGE 125, 175, Ls. 1.)

Mit dieser Entscheidung schreibt das Grundgesetz also die Verpflichtung des Staates fest, das sozio-kulturelle Existenzminimum abzusichern, mit einer Ewigkeitsgarantie. Wenn es linker Politik gelingt, die "physische Existenz« und das "Mindestmaß an Teilhabe am gesellschaftlichen und kulturellen und politischen Leben « öffentlich so zu besetzen, dass zu diesem nicht nur Leistungen zum Lebensunterhalt gehören, sondern auch Wohnraum, Zugang zu Bildung und Kultur, Mobilität, Versorgung mit Breitband und umfassende medizinische Versorgung, dann kann mit Verweis auf das Sozialstaatsprinzip im Rahmen von Gesetzgebung auch eine grundlegend andere Wirtschaftsweise umgesetzt werden. Unter Berücksich- tigung der Eigentumsgarantie des Artikel 14 GG.

Eigentumsgarantie hindert Durchsetzung des Sozialstaatsgebot nicht

Wird über die Gewährleistung des Sozialstaatsprinzips geredet, kommt schnell ein Neoliberaler oder eine Neoliberale um die Ecke und winkt mit der Eigentumsgarantie des Artikel 14 GG. Als sei dies ein Recht, das über dem Grundrecht steht, das nicht angetastet werden darf. Doch das ist nicht überzeugend. Vielmehr scheint hier eine bewusste Unkenntnis des vielfältigen Regelungsgehalts des Artikel 14 GG vorzuliegen. Der Artikel $14 \mathrm{GG}$ enthält auf der einen Seite eine Eigentumsgarantie (Gewährleistungsgarantie), auf der anderen Seite wird aber auch klar formuliert: Eigentum verpflichtet, sein Gebrauch soll dem Wohle der Allgemeinheit dienen.

Zunächst ist es so, dass ein einfaches Gesetz, nämlich das Bürgerliche Gesetzbuch (BGB), definiert was Eigentum eigentlich ist. Dieses regelt in $\mathrm{Pa}$ ragraf 903, dass der Eigentümer oder die Eigentümerin mit einer Sache nach Belieben verfahren und andere von jeder Einwirkung ausschließen kann, soweit nicht das Gesetz oder Rechte Dritter entgegenstehen. Wie aber jemand zum Eigentümer oder zur Eigentümerin wird, ist ein politischer Aushandlungsprozess, der sich in verschiedenen Gesetzen niederschlägt. Grundsätzlich kann zum Beispiel durchaus gefragt werden, warum nach dem Gesetz über Arbeitnehmererfindungen (ArbnErfG) der Arbeitnehmer oder die Arbeitnehmerin gegen ihr Unternehmen einen Anspruch auf angemessene Vergütung hat, dieses sich aber eine sogenannte 
Diensterfindung zu Eigen machen kann und das Patent erwirbt, das eine Form von Eigentum ist. Das Grundgesetz jedenfalls stellt mit Artikel 14 keine Regel auf, nach der das zwingend so sein muss. Es lohnt sich also, in der politischen Debatte zu hinterfragen, wie es eigentlich zu Eigentum kommt, um dies dann in die juristische Debatte einzubringen.

Das Grundgesetz ist also zunächst offen für die Regelung, wie Eigentum entsteht. In Verbindung mit dem Sozialstaatsprinzip eröffnet es zudem den Spielraum, den Gebrauch des Eigentums in den Dienst der Allgemeinheit zu stellen, etwas, was neoliberale Argumentationsmuster nicht erkennen (wollen). Das Bundesverfassungsgericht hat zum Beispiel im Hinblick auf die Gewährleistungsgarantie des Eigentums formuliert: "Die Gewährleistung des Rechtsinstituts wird nicht angetastet, wenn für die Allgemeinheit lebensnotwendige Güter zur Sicherung überragender Gemeinwohlbelange und zur Abwehr von Gefahren nicht der Privatrechtsordnung, sondern einer öffentlich-rechtlichen Ordnung unterstellt werden.«(BVerfGE 58, 300, Rdnr. 174). Hier sagt das Bundesverfassungsgericht nichts anderes, als dass mit staatlichen Ordnungsmitteln die Frage geregelt werden kann, was Eigentum ist und wie es verwendet werden kann, ohne dass dies die Eigentumsgarantie des Artikel 14 Grundgesetz verletzt. Diese Entscheidung findet in politischen Debatten kaum Berücksichtigung, dabei eröffnet sie die Möglichkeit, der Marktorientierung etwas entgegenzusetzen. Natürlich kann dies nicht einfach per Ordnungsrecht umgesetzt werden. Es bedarf dazu der Notwendigkeit, dass überragende Gemeinwohlbelange in Bezug auf "für die Allgemeinheit lebensnotwendiger Güter " gesichert werden müssen und/oder die Abwehr einer Gefahr. Es ist Verbalradikalismus nach Enteignung zu rufen, es ist radikaler Reformismus, die Möglichkeiten und Wege, die mit der Rechtsprechung des Bundesverfassungsgerichts aufgezeigt sind, zu nutzen. Politisch gesprochen: Wenn ausreichender Wohnraum zu bezahlbaren Preisen, eine ausreichende Versorgung von Patient*innen und die Zurverfügungstellung von Schutzkleidung über den Markt nicht realisierbar sind, dann kann dem mit Ordnungsrecht abgeholfen werden. Dazu bedarf es eines politischen Willens und einer durchdachten juristischen Untersetzung.

Das Bundesverfassungsgericht bewegt sich mit der zitierten Entscheidung in der nicht ganz so einfachen Systematik des Artikel 14 GG, die häufig verkannt wird und zu Missverständnissen führt. Denn die Entscheidung des Bundesverfassungsgerichts stellt sich am Ende als sogenannte Inhalts- und Schrankenbestimmung heraus. Als Inhalts- und Schrankenbestimmung ist definiert, was mit dem Eigentum gemacht oder nicht gemacht werden darf, ohne dass dies eine Enteignung ist oder die Gewährleistung des Eigentums nach dem Grundgesetz infrage gestellt wird Die Mehrzahl der Eingriffe in das Eigentum sind Inhalts- und Schrankenbestimmungen. Natürlich müssen auch solche Inhalts- und Schrankenbestimmungen extra begründet werden und bedürfen einer Rechtfertigung. Diese wiederum ist gegeben - darauf ist 
bereits verwiesen worden - wenn der Markt beispielsweise die Versorgung mit Wohnraum oder die Krankenversorgung nicht ausreichend garantieren kann. Allerdings nur, wenn von der Politik deutlich gemacht wird, dass dies unter Sozialstaatsgesichtspunkten zur Daseinsvorsorge gehört. Eine Gesellschaft, in der die ausreichende Versorgung von Kranken oder die Versorgung von Wohnraum nicht als Gemeinwohlbelang anerkannt wird, kann auch nicht mit dem Sozialstaatsgebot argumentieren. Es ist deshalb für die juristische Auseinandersetzung wichtig, welches Verständnis von Sozialstaatlichkeit existiert.

\section{Enteignung ist keine Lösung}

Nun erlaubt der Artikel 14 GG neben diesen Inhalts- und Schrankenbestimmungen auch eine Enteignung zum Wohle der Allgemeinheit. Das klingt unglaublich radikal, ist es aber nicht. Enteignungen können radikal sein, für emanzipatorische Politik, die auf eine Veränderung der Wirtschaftsordnung zielt, sind sie sehr überschätzt. Inhaltsund Schrankenbestimmungen sind hier häufig nachhaltiger als eine Enteignung. Insbesondere in linken Kreisen wird immer wieder die Idee einer Enteignung ins Spiel gebracht, wenn es darum geht, die Daseinsvorsorge zu gewährleisten und den Sozialstaat zu stärken. Aber: Enteignung ist keine Lösung.

Eine Enteignung liegt dann vor, wenn diese - entsprechend Artikel 14 Absatz 3 GG - durch ein Gesetz oder aufgrund eines Gesetzes erfolgt, das Art und Ausmaß der Entschädigung regelt, und die Enteignung zum Woh- le der Allgemeinheit stattfindet. Kurz gesagt: Soweit in einem Gesetz oder aufgrund eines Gesetzes keine Entschädigung festgelegt ist, ist es keine Enteignung. Das Bundesverfassungsgericht drückt dies so aus: »Die Enteignung im verfassungsrechtlichen Sinn ist auf die vollständige oder teilweise Entziehung konkreter subjektiver Eigentumspositionen im Sinne des Art. 14 Abs. 1 Satz 1 GG zur Erfüllung bestimmter öffentlicher Aufgaben gerichtet.« (BVerfGE 70, 191, Rdnr. 30)

Bei einer Enteignung wird einem Eigentümer etwas gegen eine Entschädigung wegegenommen und einem neuen Eigentümer übertragen. Bedingung ist, dass dies zur Erfüllung öffentlicher Aufgaben erforderlich ist. Die Enteignung sagt nun aber nichts darüber aus, wer diese öffentlichen Aufgaben übernimmt und ob es dabei auch um Profit gehen darf. Und das ist die Krux. Bei der Enteignung ist es möglich, einem Akteur in Privatrechtsform (Aktiengesellschaft, $\mathrm{GmbH}$ ) das Eigentum zu entziehen, und dieses dann einem anderen Akteur in Privatrechtsform zu übertragen. Dieser neue Eigentümer kann aber beispielsweise weiter mit Gewinnerzielungsabsicht wirtschaften. Die demokratische Kontrolle über das Eigentum ist nicht sicher gewährleistet. Es muss nur auch öffentlichen Aufgaben dienen. Und so finden Enteignungen auch in nicht unerheblichem Umfang statt. In diesem Zusammenhang kann auf Enteignungen im Rahmen von Infrastrukturmaßnahmen verwiesen werden, wie dem Straßen- oder Autobahnbau oder dem Bau von Versorgungsleitungen, deren Voraussetzungen in Paragraf 87 BauGB geregelt ist. Das am Ende auch dieje- 
nigen von der Enteignung partizipieren, deren finanzielle Möglichkeiten beschränkt sind, ist damit noch lange nicht sichergestellt.

\section{Vergesellschaftung ist nicht Verstaatlichung aber notwendig}

Wenn nun aber die Enteignung keine Alternative ist, kommt die Vergesellschaftung ins Spiel. Bedingt. Der Artikel 15 GG eröffnet die Option, Grund und Boden, Naturschätze und Produktionsmittel in Gemeineigentum oder andere Formen der Gemeinwirtschaft zu überführen. Bei Gemeinwirtschaft geht es um die Deckung eines öffentlichen oder gesellschaftlichen Bedarfes ohne Gewinnabsicht zur Verfolgung von Gemeinwohlzielen (von Mangoldt/Klein/ Starck 2010, Art. 15, Rdnr. 16; BeckOK, Art. 15, Rdnr. 11).

Durch die Überführung in Gemeineigentum oder andere Formen der Gemeinwirtschaft ist klar, dass das, was vergesellschaftet wird, zukünftig nicht mit Gewinnabsicht bewirtschaftet werden darf. Das wiederum dürfte der Sicherung des Sozialstaates und damit der Sicherung der Daseinsvorsorge dienen. Denn ohne Profitdruck lässt sich der Zugang zum-weit ausgelegten-soziokulturellen Existenzminimum viel besser gewährleisten. Nun weist Andreas Fisahn (2018: 60ff.) darauf hin, dass durch die EU-Verträge Wirtschaftsdemokratie erheblich erschwert, wenn nicht sogar verhindert wird. Hier erweist es sich ausnahmsweise als Vorteil, dass der Artikel 15 GG die Vergesellschaftung auf bestimmte Vergesellschaftungsobjekte beschränkt. Vergesellschaftung kann nicht die gesamte Wirtschaft erfassen. Vergesellschaftung geht nach Art. 15 GG nur für Grund und Boden (sowie sich darauf befindlichen Gebäuden), Naturschätze und Produktionsmittel. Die angesprochenen Arbeitnehmererfindungen wären demnach nicht vergesellschaftungsfähig. Im Hinblick auf Wirtschaftsdemokratie hat Fisahn also Recht, einer Vergesellschaftung hingegen dürften die EU-Verträge aus den genannten Gründen nicht entgegenstehen.

Artikel 15 GG ermöglicht lediglich für die genannten Vergesellschaftungsobjekte eine gemeinwirtschaftliche Organisation der Bewirtschaftung. Um einen breitestmöglichen Anwendungsbereich des Artikel 15 GG zu eröffnen, kommt es deshalb auf eine weite Auslegung des Begriffs Produktionsmittel an. Denn bei Wohnraum ist eine Vergesellschaftung wegen "Grund und Boden « und den sich darauf befindlichen Gebäuden möglich. Auch bei Krankenhäusern scheint mir ein solcher Ansatz nicht ausgeschlossen zu sein. Für eine digitalisierte Welt wiederum ist aber zentral, dass der Produktionsmittelbegriff beispielsweise auch Algorithmen und Patente umfasst. Eine politische Debatte darüber, wie zur Gewährleistung des Sozialstaates und damit der Daseinsvorsorge auch ein nicht klassischer Produktionsmittelbegriff zum Wohle der Allgemeinheit erfasst werden kann, wäre deshalb dringend nötig.

Dabei muss es gar nicht immer um Vergesellschaftungen gehen, sondern auch um andere Möglichkeiten. Hier sei kurz auf das Patenrecht hingewiesen. Die Idee des Patentrechts ist eigentlich, dass eine zugrundeliegende Idee offengelegt wird. Das ändert nur nichts daran, dass wegen des Eigentumsschutzes 
nicht jede*r diese Idee nutzen kann. Es findet eine Privatisierung von Wissen statt. Das Wissen kann nur begrenzt genutzt werden, weil eine Nachahmung durch ein Ausschließlichkeitsrecht zeitlich befristet ausgeschlossen wird oder nur gegen Lizenzierung (und Bezahlung) möglich ist. Die beliebte Forderung nach Abschaffung des Patentrechtes hätte zur Folge, dass die existierende Offenlegungspflicht der Idee hinter dem Patent entfällt. Das wiederum würde zu einer originären Privatisierung von Wissen führen. Nun existiert die Möglichkeit einer »Vergesellschaftung" etwa dadurch, dass Dinge, die überwiegend mit öffentlichen Mitteln erforscht und entwickelt wurden, auch für die Öffentlichkeit nutzbar sein müssen. Es wäre auch denkbar, das Regel-Ausnahme-Verhältnis bei Patenten umzukehren, das heißt es wird grundsätzlich die Nachahmung erlaubt und nur in Ausnahmefällen ausgeschlossen. Und schließlich bliebe noch die Idee eine patent buyouts. Die Idee dahinter ist, dass der Staat oder ein anderer Sponsor, zum Beispiel eine internationale Organisation, versucht, die Effizienzprobleme des Patentschutzes dadurch $\mathrm{zu}$ vermeiden, indem Patente mit besonders großen sozialen Erträgen aufgekauft und zur allgemeinen Verwendung freigegeben werden.

Doch zurück zu Artikel 15 GG. Dieser ermöglicht Sozialisierungen und eine gemeinwirtschaftliche Bewirtschaftung. Es sei aber vor einem Trugschluss gewarnt: Vergesellschaftung ist nicht per se Verstaatlichung. Und das ist gut so. Denn Gemeineigentum »bedeutet Eigentum einer kollektiven Gesamtheit und zeichnet sich dadurch aus, dass statt eines Individuums der Staat, die Gemeinden oder sonstige Selbstverwaltungseinrichtungen Träger des Eigentumsrechts sind « (BeckOK, Art.15, Rdnr. 12) Nicht nur aus historischen Gründen, sondern auch aus Gründen einer gesunden Skepsis gegen den Staat, ist immer wieder zu betonen, dass Vergesellschaftung nach Art. 15 GG es zwar zulässt, dass der Staat Träger des Eigentumsrechts wird, dies aber zum Glück nicht zwingend ist. Bei Vergesellschaftungen sollte der Fokus immer auf Selbstverwaltungseinrichtungen als Trägern des Eigentumsrechts liegen, denen staatliche Akteure zur Seite gestellt werden können. Grundsätzlich gilt: »Gemeineigentum ist von der Verstaatlichung zu unterscheiden, da es nicht ausreicht, Eigentum in Händen des Staates zu begründen, sondern es muss auch die Form des Wirtschaftens geändert werden.« (BeckOK, Art.15, Rdnr. 12)

\section{Literatur}

BVerfGE, Entscheidungen der amtlichen Sammlung des Bundesverfassungsgerichts (BVerfGE). URL: https://www.bundesverfassungsgericht.de/entscheidungen.

Dreier, Horst (Hg) (2016): Grundgesetz-Kommentar, 3 Bde. Tübingen.

Fisahn, Andreas (2018): Wirtschaftsdemokratie - verfassungsrechtliche Schranken und Möglichkeiten. In: Demirović, Alex (Hg.): Wirtschaftsdemokratie neu denken. Münster: 44-65.

BeckOK, Beck'scher Online-Kommentar Grundgesetz (2019), hgg. von Epping, Volker / Hillgruber, Christian. München. URL: https:// beck-online.beck.de/.

von Mangoldt, Hermann / Klein, Friedrich / Starck, Christian (Hg.) (2010): Kommentar zum Grundgesetz: GG, 3 Bde. München. 